\title{
Pkd1 and Wnt5a genetically interact to control lymphatic vascular morphogenesis in mice
}

Tevin CY. Chau ${ }^{1}$, Sungmin Baek ${ }^{1}$, Baptiste Coxam ${ }^{1}$, Renae Skoczylas ${ }^{1}$, Maria Rondon-Galeano ${ }^{1,2}$, Neil I. Bower ${ }^{1}$, Elanor N. Wainwright ${ }^{1}$, Steven SA. Stacker ${ }^{3,4,5}$, Helen M. Cooper ${ }^{6}$, Anne K. Lagendijk ${ }^{1}$, Natasha L. Harvey ${ }^{7}$, Mathias François ${ }^{1,8}$, Benjamin M. Hogan ${ }^{1,2,6,9, *}$

${ }^{1}$ Division of Genomics of Development and Disease, Institute for Molecular Bioscience, The University of Queensland, St Lucia, Queensland 4072 Australia

${ }^{2}$ Organogenesis and Cancer Program, Peter MacCallum Cancer Centre, Melbourne, VIC 3000, Australia

${ }^{3}$ Tumour Angiogenesis and Microenvironment Program, Peter MacCallum Cancer Centre, 305 Grattan St., Melbourne, Victoria 3000, Australia.

${ }^{4}$ Department of Surgery, Royal Melbourne Hospital, The University of Melbourne, Parkville, Victoria, Australia, 3050.

${ }^{5}$ Sir Peter MacCallum Department of Oncology, University of Melbourne, Melbourne, VIC 3010, Australia

${ }^{6}$ The Queensland Brain Institute, The University of Queensland, St Lucia, QLD 4072 Australia

${ }^{7}$ Centre for Cancer Biology, University of South Australia and SA Pathology, Adelaide, SA, Australia

${ }^{8}$ David Richmond Laboratory for Cardiovascular Development; Gene Regulation and Editing Program, Centenary Institute, New South Wales 2050, Australia

${ }^{9}$ Department of Anatomy and Neuroscience, University of Melbourne, Melbourne, VIC 3000, Australia

*Author for correspondence:

Professor Ben Hogan

Organogenesis and Cancer Program,

Peter MacCallum Cancer Centre,

Melbourne, VIC 3000, Australia

E-mail: ben.hogan@petermac.org

Keywords: Polycystin 1, PC1; WNT5A; planar cell polarity; lymphangiogenesis, vascular, Polycystic kidney disease 


\section{Abstract}

Lymphatic vascular development is regulated by well-characterised signalling and transcriptional pathways. These pathways regulate lymphatic endothelial cell (LEC) migration, motility, polarity and and morphogenesis. Canonical and non-canonical WNT signalling pathways are known to control LEC polarity and development of lymphatic vessels and valves. PKD1, encoding Polycystin-1, is the most commonly mutated gene in polycystic kidney disease but has also been shown to be essential in lymphatic vascular morphogenesis. The mechanism by which $P k d 1$ acts during lymphangiogenesis remains unclear. Here we find that loss of non-canonical WNT signalling components Wnt5a and Ryk phenocopy lymphatic defects seen in $P k d 1$ knockout mice. To investigate genetic interaction, we generated $P k d 1 / W n t 5 a$ double knockout mice. Loss of Wnt5a suppressed phenotypes seen in the lymphatic vasculature of $\mathrm{Pkd1}^{-/}$mice and Pkd1 deletion suppressed phenotypes observed in $\mathrm{Wnt} 5 \mathrm{a}^{-/}$ mice. Thus, we report mutually suppressive roles for $P k d 1$ and $W n t 5 a$, with developing lymphatic networks restored to a more wild-type state in double mutant mice. This genetic interaction between Pkd1 and the non-canonical WNT signalling pathway ultimately controls LEC polarity and the morphogenesis of developing vessel networks. Our work suggests that $P k d 1$ acts at least in part by regulating non-canonical WNT signalling during the formation of lymphatic vascular networks. 


\section{Introduction}

The lymphatic vasculature develops progressively in a process that involves cell specification, cell migration, cell proliferation and morphogenesis (reviewed in Koltowska et al., 2013). In mice, precursor cells along the embryonic veins acquire lymphatic endothelial cell (LEC) fate via the activity of key transcription factors (Francois et al., 2008; Oliver and Srinivasan, 2010; Wigle and Oliver, 1999). The specified LECS sprout out of the veins from approximately 10 days post coitum (dpc) and migrate throughout the embryo to form the lymphatic network (Hägerling et al., 2013; Yang et al., 2012). Early lymphatic sprouting is known to be induced by vascular endothelial growth factor C (VEGFC), signalling through its receptor VEGFR3 (Karkkainen et al., 2004). Subsequent to sprouting, LECs organise, polarise and undergo coordinated cellular and tissue morphogenesis events before forming mature lymphatic vessel networks. These later cellular events controlling vessel morphogenesis and maturation remain to be fully understood.

A growing body of evidence has demonstrated that LEC polarisation and lymphatic vascular morphogenesis can be regulated by WNT signalling components and pathways in mice (Buttler et al., 2013; Cha et al., 2016a; Cha et al., 2018; Lutze et al., 2019; Tatin et al., 2013). The non-canonical WNT/planar cell polarity (PCP) pathway is a highly conserved pathway that coordinates cell and organelle orientation, coordinated cell movements and tissue polarity during development. The PCP pathway is activated by the binding of several WNT ligands (including WNT4, WNT5A, WNT5B, WNT11 and WNT11B), to Frizzled (FZD) receptors and co-receptors; Receptor tyrosine kinase (RYK) and Rarrelated orphan receptor (ROR) (Keeble et al., 2006; Lu et al., 2004; reviewed in Roy et al., 2018). Upon activation, various PCP core components, such as Van gogh-like (VANGL) and Cadherin EGF seven-pass G-type receptor (CELSR), are recruited to the activated receptors. These proteins in turn recruit downstream effectors, such as Dishelvelled (DVL), Diversin (ANKRD6), and Prickle (PK), and generate asymmetry to establish cell polarity. Downstream, activation of factors such as RHO kinase or RAC1 influences cell migration and cell motility to co-ordinate tissue morphogenesis (reviewed in Butler and Wallingford, 2017). Canonical WNT signalling occurs when WNT ligands bind to their cognate receptors, initiating the downstream interactions that lead to translocation of B-catenin to the nucleus. This leads to downstream transcription driven by T-cell factor dependent transcription factors (TCFs) (reviewed in Cadigan and Waterman, 2012).

The autosomal dominant polycystic kidney disease (ADPKD) gene Polycystic kidney disease (Pkd1), encodes the protein Polycystin-1 (PC1), that is mutated in as many as $85 \%$ of patients with polycystic kidney disease (Peters and Sandkuijl, 1992). PC1 is a large 11 transmembrane protein with a long N- 
terminal extracellular region made up of multiple sub-domains and shown to play a role in mechanosensation (Hughes et al., 1995; Nauli et al., 2003; Nims et al., 2003). PC1 localises to the primary cilium, apical membranes and the desmosomes in various cell types and its C-terminal tail binds to Polycystin-2 (PC2 encoded by Pkd2) (Nauli et al., 2003; Yoder et al., 2002). PC2 is a calcium channel that is activated via PC1 function and influences downstream intracellular signalling events (Hanaoka et al., 2000; Nauli et al., 2003; Vassilev et al., 2001). PC1 has been reported to bind directly to core components of the non-canonical WNT/PCP pathway, PAR3 and APKC, and to regulate cell polarity in kidney epithelia. This role in kidney tubules, regulates the normal intercalation of epithelial cells, tubular extension and morphogenesis (Castelli et al., 2013). PC1 can also influence $\mathrm{Ca}^{2+}$ dependent canonical WNT signalling and in Xenopus embryos $P k d 1$ genetically interacts with Wnt9a and Dishevelled 2 (Kim et al., 2016).

We and others have found that mice mutant for $P k d 1^{-1}$ display defects in lymphatic vascular morphogenesis (Coxam et al., 2014; Outeda et al., 2014). Pkd1\% mice display prominent oedema at $14.5 \mathrm{dpc}$ that is typical in animals with lymphatic vascular defects. In the developing dermal lymphatic network, $P k d 1 \%$ mice exhibit reduced lymphatic vessel branching and dilated/distended lymphatics as a result of the failure of endothelial cells within these vessels to polarise correctly in the direction of vessel sprouting (Coxam et al., 2014; Outeda et al., 2014). Knockdown of $P k d 1$ in cultured lymphatic endothelial cells also results in a loss of polarity during cell migration (Outeda et al., 2014). These earlier studies clearly demonstrated an unexpected role for the polycystic kidney disease gene $P k d 1$ in lymphangiogenesis and identified it as a potential lymphatic disease gene. However, the precise pathway that $P k d 1$ interacts with to control lymphatic vessel development has remained unexplored.

Here, we aimed to better understand the mechanism by which $P k d 1$ regulates LEC polarity and lymphatic vascular morphogenesis. We first investigated the endothelial cell-autonomous role of $P k d 1$ in valve development, which is known to be controlled by components of the canonical and noncanonical WNT/PCP pathways. We uncovered a requirement for Pkd1 for normal lymphatic valve development. We then explored the roles of the non-canonical WNT/PCP components Wnt5a and Ryk in lymphatic development and uncovered phenotypes in knockout mice that were strikingly similar to Pkd1 mutant phenotypes. Finally, we generated Pkd1/Wnt5a double knockout mice and found that these factors genetically interact, being genetic suppressors of each other (a mutually suppressive relationship), during the regulation of LEC polarity, organisation of endothelial cells within developing vessels and in lymphatic vascular morphogenesis. Overall, these genetic studies suggest a role for $P k d 1$ in the modulation of non-canonical WNT/PCP pathway signalling in lymphatic vascular development, 
likely similar to the developmental role of Pkd1 in the morphogenesis of kidney tubules during development.

\section{$\underline{\text { Results }}$}

\section{Pkd1 cell-autonomously regulates lymphangiogenesis and lymphatic valve development}

We have previously reported a cell-autonomous role for $P k d 1$ in lymphangiogenesis using Sox18:GFPCre-ErT2(GCE) to generate conditional Pkd1 knockout mice (Coxam et al., 2014). In the previous study, Sox18:GFP-Cre-ErT2(GCE);B6.129S4-Pkd1 $1^{\mathrm{tm} 2 G g g} / J\left(P k d 1^{f / f}\right)$ mice showed subcutaneous lymphatic network defects but Tie2:Cre;Pkd1/ff mice did not. To further validate previous findings, we here used Cdh5:Cre-ErT2, (which shows activity from 7.5dpc to adult vasculature (Alva et al., 2006; Sörensen et al., 2009)) and generated Cdh5:CreERT2;Pkd1 f/f mice (hereafter referred to as $P k d 1^{i E C K O}$ ). During development, Cre activity was induced with serial tamoxifen injections at $9.5 \mathrm{dpc}, 10.5 \mathrm{dpc}$ and $11.5 \mathrm{dpc}$. At 14.5dpc, we examined subcutaneous lymphatics for Prospero homeobox 1 (PROX1), Neuropilin 2 (NRP2) and Endomucin (EMCN) expression. Pkd1 $1^{i E C K O}$ embryos showed no marked subcutaneous oedema or morphological changes compared with their wild type siblings (Figure 1A-B). The subcutaneous blood vessel network was unaffected (Figure 1 $\mathbf{C}^{\prime} \mathbf{-} \mathbf{D}^{\prime}, \mathbf{K}-\mathbf{L}$ ). However, the lymphatic network of $P k d 1^{i E C K O}$ embryos showed reduced complexity with fewer branch points and loops (Figure 1C-D, G-H). In the developing dermis, where lymphatics migrate from lateral locations towards the embryonic midline (James et al., 2013), the lymphatic sprouts of the Pkd1 $1^{i E C K O}$ embryos showed reduced medial migration and the lymphatic vascular front displayed a greater distance to the midline (Figure 1I). Furthermore, the dermal lymphatic sprouts were broader and showed a blunt-tipped morphology (Figure 1E and F). Combined with our previous work (Coxam et al., 2014), this confirmed that PC1 functions in an endothelial cell-autonomous manner to regulate lymphatic vascular morphogenesis in the developing dermis. At the level of individual cells within developing vessels, the nuclei of LECs in $P k d 1^{i E C K O}$ embryos displayed reduced ellipticity compared with those of control vessels (Figure 1E, F and J). Reduced nuclear ellipticity is an indication of cells with reduced motility and a failure to polarise (Hägerling et al., 2013), suggesting a reduction in polarity or motility in $P k d 1^{i E C K O}$ lymphatic endothelial cells.

To further study the role of PC1 in LEC polarity, we examined the formation of valves in the dermal lymphatic vasculature. The formation of dermal lymphatic valves requires the local up-regulation of Prox1 in valve-forming territories, the polarisation and the reorientation of developing LECs. In $16.5 \mathrm{dpc}$ dermal lymphatics, we observed a marked reduction in valve forming territories in $P k d 1^{i E C K O}$ embryos, as determined by scoring regions of high Prox1 expression in the dermal network relative to 
the number of network branch points (Figure $\mathbf{1 M - N ,} \mathbf{0}$ ). We observed that the nuclei of the few lymphatic valves that did form in $P k d 1^{i E C K O}$ dermal lymphatics had reduced ellipticity compared with control embryos, indicative of reduced cell polarity in valve forming territories (Figure $\mathbf{1} \mathbf{M}^{\mathbf{\prime}}, \mathbf{N}^{\mathbf{\prime}}$ and $\mathbf{Q}$ ). Quantitatively, there were fewer polarised valves in $16.5 \mathrm{dpc} P k d 1^{i E C K O}$ dermal lymphatics than in control embryos (Figure 1P) and LEC nuclei failed to reorient perpendicular to the vessel walls.

To examine how PC1 regulates lymphatic valve formation in the mesentery, where valve formation has been better characterised (reviewed in Koltowska et al., 2013; and Kume, 2015; Sabine et al., 2018), we injected tamoxifen at $15.5 \mathrm{dpc}, 16.5 \mathrm{dpc}$, and $17.5 \mathrm{dpc}$ and examined mesenteric lymphatics in $18.5 \mathrm{dpc}$ embryos. In wild type siblings, valve LEC nuclei were polarised perpendicular to the lymphatic vessels with actin stress fibres aligned at the lymphatic valves in a stereotypical pattern (Figure 1R and $\mathbf{R}^{\prime \prime}$ ). The $P k d 1^{i E C K O}$ valve LEC nuclei were identifiable based on high levels of Prox1 expression and formed an initial ring, however the cells within these valve territories failed to orient perpendicular to the lymphatic vessel (Figure 1S-S"), and had less elongated nuclei (Figure 1T). Actin recruitment or organisation at the lymphatic valves was also reduced in $P k d 1^{i E C K O}$ embryos (Figure 1R" and $\left.\mathbf{S}^{\prime \prime}\right)$, as shown by a reduced ratio of valve-to-vessel actin fluorescent intensity (Figure 1U). Thus, PC1 endothelial cell-autonomously regulates the formation of lymphatic valves in the mesentery. The lymphatic valve phenotypes that we observed here and the randomised Golgi orientation in the dermal lymphatic sprouts previously reported (Coxam et al., 2014) strongly suggested a loss of cell polarity in $P k d 1$ mutants. The lymphatic valve phenotypes were also similar to valve phenotypes seen in published mouse models such as the Fat4 conditional knockout mouse or the Celsr1 mutant mouse, both impacting non-canonical WNT/PCP signalling mesenteric lymphatic valves (Betterman et al., 2020; Tatin et al., 2013).

The planar cell polarity pathway ligand WNT5A regulates lymphatic endothelial cell polarity during development

The LEC polarity phenotypes observed in $P k d 1^{i E C K O}$ and the previously described interaction between PC1 and PCP components in epithelial cells led us to further investigate the relationship between PC1 and the non-canonical WNT/PCP pathway using genetics. Previous studies have shown that a PCP ligand, WNT5A, is required for dermal lymphatic development (Buttler et al., 2013; Lutze et al., 2019). These studies described lymphatic sprout morphology and structure in Wnt5a $a^{-1}$ embryos but polarity phenotypes in lymphatic network morphogenesis were not fully described.

We analysed the dermal lymphatics of $W n t 5 a^{-1-}$ embryos at $13.5 \mathrm{dpc}$ and observed reduced network formation in the $W n t 5 a^{-1-}$ dermal lymphatics (Figure 2A-B). Wnt5a $a^{-1-}$ dermal lymphatics were dilated 
and the lymphatic sprouts failed to migrate towards the midline of the embryo (Figure 2C-D). In Wnt5 $a^{-/}$mice, there was an increased number of cells in lymphatic vessels (measured along $150 \mu \mathrm{m}$ of the vessel starting at the leading vessel tips of lymphatic sprouts) and the LEC nuclei displayed reduced ellipticity compared with controls (Figure 2E-F). This suggested reduced cell polarity and a lack of LEC migration. To assess whether this phenocopies the Pkd1-knockout mice, we investigated LEC polarity more directly. In polarised endothelial cells, the Golgi aligns in the direction of migration, positioning between the nucleus and the direction of migration. Lymphatic vascular mutants with disrupted polarity show a loss of this nuclear-Golgi polarisation in the direction of the embryonic midline (Betterman et al., 2020; Coxam et al., 2014). We examined the polarity of LECs in Wnt5 $a^{-/}$ embryos by examining Golgi (GOLPH4) localisation relative to the LEC nuclei (PROX1) and the midline (Figure 2I-J). The Golgi body aligned in the direction of migration in wild type sprouts, but this was reduced in Wnt5a $a^{-1-}$ mice (Figure $2 \mathrm{~K}-\mathrm{L}$ ), indicating a loss of cell polarity in Wnt5a ${ }^{-1-}$ LECS. These phenotypes were in line with the report from Lutze et al. (2019), and further suggest that the loss of PCP signalling, polarity and convergence extension leads to dilated lymphatic vessels and isolated lymphatic cysts in Wnt5a $a^{-/}$mice. The blood vessel network also had abnormal endothelial cell clusters along the midline (Figure $\mathbf{2} \mathbf{A}^{\prime}-\mathbf{B}^{\prime}$ ) but there were no measurable differences in the number of branch points and loops in blood vessel networks at $13.5 \mathrm{dpc}$, a stage when wild type embryos were yet to form a dense blood vessel network spanning the embryonic midline (Figure $\mathbf{2 G - H}$ ). Overall, these phenotypes suggested that WNT5A, a PCP pathway ligand, regulates lymphangiogenesis by controlling cell polarity, displaying a similar but more severe phenotype than $P k d 1^{i E C K O}$ mice.

\section{The planar cell polarity co-receptor Ryk regulates lymphangiogenesis during development}

During PCP signalling, WNT5A binds to receptors, including FZD, ROR and RYK, to initiate downstream cellular processes (reviewed in Butler and Wallingford, 2017). WNT5A enhances RYK and VANGL2 binding to establish cell polarity (Andre et al., 2012). RYK and VANGL2 regulate PCP during mouse cochlea and neural tube development (Macheda et al., 2012). To further understand the role of PCP pathway components in lymphatic development, we assessed the role of the co-receptor RYK in lymphangiogenesis. Ryk knockout mice display classical PCP pathway phenotypes that include craniofacial and neural tube defects, and misoriented stereociliary bundles of sensory hair cells in the cochlea (Halford et al., 2000; Macheda et al., 2012). Upon examination of the dermal lymphatics, $R y k$ /- embryos showed similar but milder lymphatic defects compared with Wnt5a/- mice. The Ryk ${ }^{-/}$ mutant dermal lymphatic network had reduced branching and numbers of vascular loops at $14.5 \mathrm{dpc}$ (Figure 3A-B), lymphatic sprouts displayed a stereotypical blunt-tipped and widened morphology (Figure 3C-D, E) and there were increased numbers of cells in each vessel normalised to vessel length, 
scored as cell number per $100 \mu \mathrm{m}$ of vessel from the tips of lymphatic sprouts (Figure 3F). The blood vessel network was not affected in these mutants (Figure $3 \mathbf{A}^{\prime}-\mathbf{B}^{\prime}, \mathbf{G}-\mathbf{H}$ ). Overall, this indicates that RYK, a WNT5A co-receptor, is also a regulator of lymphatic vascular morphogenesis during development.

\section{$P k d 1$ and Wnt5a genetically interact to control lymphatic endothelial cell polarity and lymphatic} vessel morphogenesis of the dermal vasculature

Given similar phenotypes in the absence of $P k d 1, W n t 5 a$ and $R y k$, we hypothesised that $P k d 1$ may modulate lymphatic vascular morphogenesis by interacting with the non-canonical WNT/PCP signalling pathway. We used genetics to probe for an interaction at the level of vessel phenotype. We generated $P \mathrm{kd1}^{+/-}$; Wnt5 $\mathrm{C}^{+/-}$double heterozygous mice (using a ubiquitous Pkd1-knockout model, not the iECKO model) and in-crossed the double carriers to examine the lymphatic networks for the various genotypes generated. We used a number of quantitative phenotypic measurements to analyse the dermal lymphatics of the $14.5 \mathrm{dpc}$ embryos.

As reported previously, both $P k d 1 \%$ and $W n t 5 a^{-/}$lymphatic networks had fewer branch points and loops (Figure 4A-C), and the lymphatic vessels were widened (Figure 4G-I', M). We found no evidence that loss of additional copies of Wnt5a modified Pkd1 mutant phenotypes at the level of vessel branch points (Figure 4B, D-E). We also found no evidence that loss of additional copies of Pkd1 modified phenotypes at the level of vessel branch points in Wnt5a mutants (Figure 4C, D, F). However, an examination of lymphatic vessel morphology at the level of vessel width, revealed that developing dermal lymphatics of $\mathrm{Pkd1}^{-}$; $\mathrm{Wnt}_{\mathrm{Na}} \mathrm{a}^{+/}$embryos were thinner than those in $\mathrm{Pkd1} 1^{-}$knockouts, identifying Wnt5a as a dominant suppressor of the $P k d 1$ lymphatic vessel width phenotype (Figure $4 \mathrm{~J}$ K, M). Furthermore, developing dermal lymphatics of $P k d 1^{-} ;$Wnt5 $a^{-/}$double mutants were also thinner than in $\mathrm{Pkd1}^{-}$or Wnt5 $\mathrm{a}^{-/}$single mutant mice (Figure $4 \mathrm{I}-\mathrm{L}$ ). At the level of LECs per vessel (within $100 \mu \mathrm{m}$ from the tips of lymphatic sprouts), $P \mathrm{kd1}^{-1} ; \mathrm{Wnt} 5 \mathrm{a}^{- \text {- }}$ embryos displayed fewer cells per vessel than in $\mathrm{Pkd1}^{-}$embryos and were therefore closer to a wild type control vessel (Figure $4 \mathrm{~N}$ ). As these data may suggest a genetic interaction at the level of cell polarity and migration, we further

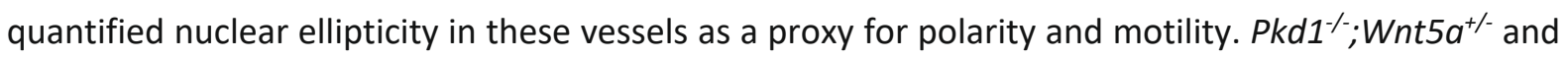
$P k d 1^{-1}$; Wnt $5 a^{-/-}$LEC nuclei were more elliptical than those quantified in the $P k d 1^{-\%}$ LEC nuclei (Figure 40). Finally, we assessed the number of LECs at the tips of leading vessels, as a measure of the blunt tip phenotype. Here we saw not just that loss of alleles of Wnt5a suppressed (or partially rescued) the $P k d 1$ phenotype, but that loss of a single copy of $P k d 1$ dominantly suppressed the Wnt5a phenotype as well (Figure 4P). 
We examined the blood vessel network for similar genetic interaction. The blood vessel network had a reduced number of branch points in all mutant genotypes when compared with wild type embryos in medial and flank regions, likely due to subcutaneous oedema in these mutant embryos. However, there was no change or rescue of this phenotype in the double mutants (Figure $4 A^{\prime}-C^{\prime}, Q$ ). This suggests that the genetic interaction described above is lymphatic restricted and blood vessel phenotypes are likely to be influenced by the sub cutaneous oedema that has been previously reported. We also examined the blood vessel networks between the various genotypes for numbers of loops but found no significant changes compared with wild type (Figure $4 A^{\prime}-C^{\prime}, Q$ ).

Overall, this genetic interaction in lymphatic network morphogenesis between Pkd1 and Wnt5a led to partial phenotypic rescue in various genotypes and provides genetic evidence that $P k d 1$ and Wnt5a regulate cell polarisation and vessel morphogenesis during lymphangiogenesis by acting in the same genetic pathway.

\section{Discussion}

In this study, we show that $P k d 1$, Wnt5a and Ryk are important for lymphangiogenesis and LEC polarity. Pkd1 regulates subcutaneous lymphatic network and valve formation cell-autonomously. The cell migration, elongation and orientation defects reported here and in earlier studies (Coxam et al., 2014; Outeda et al., 2014) suggest that $P k d 1$ regulates lymphangiogenesis through controlling LEC polarity. WNT5A and RYK also regulate lymphangiogenesis, with reduced lymphatic network complexity in Ryk and Wnt5a mutants and loss of polarised Golgi body alignment and reduced nuclei elongation in Wnt5a mutants. To further probe the relationship between Pkd1 and the non-canonical WNT/PCP ligand WNT5A, we generated double mutants and revealed a clear genetic interaction between Pkd1 and $W n t 5 a$. The loss of Wnt5a partially rescued (suppressed) the dermal lymphatic phenotypes caused by the loss of $P k d 1$, and in the case of the morphogenesis of vessel tips, vice versa. Overall, these findings report new phenotypes in Pkd1, Wnt5a and Ryk knockout mice and suggest that PC1 acts in the PCP pathway during lymphatic development.

Several studies have suggested the importance of the PCP pathway in lymphangiogenesis. FAT4, a component of the global PCP modules, is required for LEC polarisation and lymphatic development in mice and mutated in the inherited lymphoedema syndrome - Hennekam syndrome (Alders et al., 2014; Betterman et al., 2020). Lymphatic specific deletion of Fat4 in mice results in lymphatic vessel networks with reduced branch points and increased vessel width. It also leads to reduced mesenteric lymphatic valve formation as does the loss of its ligand Dachsous1 (Betterman et al., 2020; Pujol et al., 
2017). LECs in lymphatic specific Fat4 deletion mice are more rounded, and their Golgi bodies less aligned in the direction of migration and the nuclei (Betterman et al., 2020). Overall, these phenotypes are strikingly similar to those observed in Pkd1 knockout mice. WNT5A, a ligand of the PCP pathway, is also required for dermal lymphatic morphogenesis (Buttler et al., 2013; Lutze et al., 2019). Wnt5anull mice have reduced numbers of dermal lymphatic capillaries but no difference in LEC proliferation (Buttler et al., 2013). Lymphatic vessels in Wnt5a-null mice are cyst-like, non-functional and bloodfilled (Lutze et al., 2019). We examined these vessels at $13.5 \mathrm{dpc}$ rather than $14.5 \mathrm{dpc}$ in single mutant animals because of severe morphological defects in these mutants at later stages. These lymphatic vessels show a more severe but still similar phenotype to Pkd1 knockout mice. In the context of lymphatic valve development, the PCP core components VANGL2 and CELSR1 control valve LEC polarisation and orientation in the mesentery (Tatin et al., 2013). Coupled with our findings above these similar observations support an important role for the non-canonical WNT/PCP pathway in lymphatic network formation and LEC polarity and a role for $P k d 1$ interacting with this pathway.

The question remains, how does PC1 interact with the PCP pathway at a mechanistic level? PC1 (encoded by Pkd1) can directly interact with PCP components. PC1 complexes with PAR3 to promote PAR3/aPKC complex formation in mouse embryonic fibroblasts (MEFs) and so it is possible the interaction is also direct in LECs (Castelli et al., 2013). However, PC1 also complexes with PC2 to form a $\mathrm{Ca}^{2+}$ channel and WNT5A can bind and activate the PC1/PC2 complex to induce $\mathrm{Ca}^{2+}$ influx (Kim et al., 2016). It is currently unclear whether WNT/Ca ${ }^{2+}$ signalling contributes to lymphatic development, although it has been shown that shear stress induces $\mathrm{Ca}^{2+}$ pulses in LECs (Choi et al., 2017b; Surya et al., 2019) and the $\mathrm{Ca}^{2+}$ channel ORAI1 is required for trachea and dermal lymphatic development (Choi et al., 2017a). So it is possible that PC1 may influence Ca2+-dependent WNT signalling, or PCP signalling, or both, to control vessel morphogenesis and the genetic data presented here does not currently distinguish between these possible mechanisms. Of note, canonical WNT signalling is also required for lymphatic development (Cha et al., 2018). The deletion of 6 -catenin and the WNT coreceptors $L r p 5$ and $L r p 6$ from lymphatics leads lymphatic morphogenesis defects in the dermal vessel network and lymphatic valve formation defects (Cha et al., 2016b; Cha et al., 2018). Thus, there appear to be multiple ways in which WNT signalling cascades can influence lymphatic development and the precise role (or roles) of $P k d 1 / P C 1$ will require further study in the future.

$P k d 1$ is the most commonly mutated gene in autosomal dominant polycystic kidney disease. As such, most emphasis on the role of $P k d 1$ in disease has focussed on the kidney. However, this work and our earlier findings suggest that $P k d 1$ is a candidate that could influence diseases of the lymphatic vasculature. Of note, in kidney development $P k d 1$ also acts by interacting with WNT pathways and the 
regulation of cell polarity, so there may be common mechanisms at play in both contexts. If PC1 does act via similar mechanisms to FAT4 and DCHS1, given that Fat4 that is mutated in Hennekam syndrome, it will be interesting to explore the interaction between Fat4 and Pkd1 and determine if $P k d 1$ may modify disease phenotypes in lymphoedema. It will also be interesting to know if RYK or WNT5A mutations are associated with primary lymphoedema in humans. Overall, this study confirmed the roles of PC1, WNT5A and RYK in regulating LEC polarity, and identified additional steps of lymphangiogenesis controlled by $P k d 1$ (valve formation in the dermis and mesentery). We found that $P k d 1$ and $W n t 5 a$ genetically interact and suggest that $P k d 1$ regulates LEC polarity by influencing the PCP pathway. This work thus provides a deeper understanding of the role of $P k d 1$ in lymphatic vascular development and points to potential roles in lymphatic disease. 


\section{Materials and methods}

\section{Animals}

$P k d 1^{f / f} ; C d h 5-c r e / E R T 2$ mice were generated by crossing B6.129S4-Pkd1 $1^{\text {tm2Ggg }} / J\left(P k d 1^{f / f}\right.$; MGI:97603) mice to $\mathrm{Tg}(\mathrm{Cdh5}$-cre/ERT2)1Rha (MGI:3848982), and breeding carriers to generate subsequent generations and experimental animals. The breeding strategy for the $P k d 1^{+-}$mouse model used in this study was previously described in Coxam et al. (2014). Wnt5a ${ }^{-/}$mice were generated by in-crossing B6;129S7-Wnt5a $a^{\text {tm1Amc }} / J$ (mixed background; MGI:98958). $P k d 1^{+/-}$and $W n t 5 a^{+/-}$were crossed to generate double heterozygous carriers. The carriers were incrossed to produce various genotypes of $P k d 1$; Wnt5a double mutants and littermate controls.

Timed pregnancies were set up and adult female mice checked for plugs accordingly. To induce Cre recombination and after pregnancy is confirmed, the female is given a dose of 4Hydroxytamoxifen (Sigma-Aldrich Cat\# H6278) dissolved in 90\% sunflower oil/10\% Ethanol at a concentration of $10 \mathrm{mg} / \mathrm{mL}$, during three consecutive days. Dosing of $40 \mathrm{mg}$ per $\mathrm{kg}$ of body weight was given by intraperitoneal injection. To excise the floxed alleles in $P k d 1^{f / f} ; C d h 5-c r e / E R T$ for the dermal lymphatic model, pregnant females were injected at $9.5 \mathrm{dpc}, 10.5 \mathrm{dpc}$, and $11.5 \mathrm{dpc}$ and, for the mesenteric lymphatic model pregnant females were injected at $15.5 \mathrm{dpc}, 16.5 \mathrm{dpc}$ and $17.5 \mathrm{dpc}$.

Genotyping primer sequences: $P k d 1^{f l / f l}$-geno Forward-5'-CCGCCTTGCTCTACTTTCC-3'; Pkd1 1/ffl-geno Reverse-5'-AGGGCTTTTCTTGCTGGTCT-3'; Cre-geno Forward-5'CTGACCGTACACCAAAATTTGCCTG-3'; Cre-geno Reverse- 5'GATAATCGCGAACATCTTCAGGTTC-3'; Pkd1-geno Forward-5'-CCTGCCTTGCTCTACTTTCC-3'; Pkd1-geno Reverse-5'-TCGTGTTCCCTTACCAACCCTC-3'; Wnt5a-geno Forward- 5'GTTGATTCTGTGTGCCTATTCTGC-3'; Wnt5a-geno Reverse1-5'-CCCCGTGGAACTGAGTGTAT3'; Wnt5a-geno Reverse2-5'-TGGA TGTGGAA TGTGTGCGAG-3'. The Ryk knock-out mouse breeding and genotyping genotyping was as previously described in Halford et al. (2000).

\section{Immunofluorescence staining}

Embryos were harvested at $14.5 \mathrm{dpc}$ or $16.5 \mathrm{dpc}$ and fixed in $4 \%$ paraformaldehyde in phosphate buffered saline (PBS) at $4^{\circ} \mathrm{C}$ overnight and then stored in PBS. Dorsal skins from under ear shell to above hind limb were dissected out from the back of embryos and muscle 
tissue was removed from the skin as previously described by James et al. (2013). The mesenteric membrane was isolated from intestines when harvesting embryos at $18.5 \mathrm{dpc}$. The mesenteric membrane was fixed in $4 \%$ paraformaldehyde in PBS at room temperature for 1 hour, and stored in PBS. The skins and mesenteric membranes were blocked in $10 \%$ heat-inactivated horse serum in washing buffer containing 100mM maleic acid, 1\% dimethyl sulfoxide and $0.1 \%$ Triton-X in PBS for 1 hour at room temperature. After blocking, samples were incubated in blocking buffer with primary antibodies overnight at $4^{\circ} \mathrm{C}$. Samples were then washed during the day with washing buffer for 5 hours and then incubated in blocking buffer with secondary antibodies. Samples were washed again with washing buffer throughout the day and mounted with Vectashield Antifade Mounting Medium (Vector Laboratories Cat\# H-1000-10) on a clear microscope slide. Various combinations of antibodies were used to achieve co-staining of different proteins. Primary antibodies used were: anti-EMCN (Santa Cruz SC-53941), anti-PROX1 (AngioBio 11-002), anti-NRP2 (R\&D System AF567). The following secondary antibodies were used: Donkey anti-Goat $\lg G(H+L)$ Cross-Adsorbed, Alexa Fluor 488 (Invitrogen, A11055), Goat anti-Rat IgG (H + L) CrossAdsorbed, Alexa Fluor 647 (Invi-trogen, A21247), Donkey anti-Rabbit IgG (H + L)Highly CrossAdsorbed, Alexa Fluor 594 (Invitrogen, A21207), Alexa Fluor 488 Phalloidin (Invitrogen, A12379). Stained skins and mesenteric membranes were mounted on glass slides with gelvatol mounting medium and stored at $4^{\circ} \mathrm{C}$ until imaging.

\section{Imaging}

All mouse embryos were imaged prior to fixation using a Leica M165 FC at $0.73 x$ magnification, illuminated by an external light source. The mounted skins of Figure 1 and 4 were imaged on a Nikon Ti-E Deconvolution Microscope with a 4x Plan Apochromat N.A. 0.2 objective to generate skin scan images for quantifications of lymphatic network formation. The mounted skins and mesenteries of Figure 1 and 4 were imaged on a Zeiss LSM 710 Meta Confocal Scanner with 40x Oil Plan Apochromat N.A. 1.3 and 63x Oil Plan Apochromat N.A. 1.4 objectives to generate vessel images for quantifications all cellular level. The mounted skin of Figure 3 and 4 were imaged with a Zeiss LSM 510 or Zeiss LSM 710 Meta Confocal Scanner with 40x Oil Plan Apochromat N.A. 1.3 and 63x Oil Plan Apochromat N.A. 1.4 objectives. 


\section{Quantification and image analysis}

All images were analysed with Fiji (Schindelin et al., 2012). We quantified all branch points and loops of the subcutaneous lymphatic network within $2000 \mu \mathrm{m}$ from the midline in $13.5 \mathrm{dpc}$ and $14.5 \mathrm{dpc}$ embryos. We randomly selected 5 lymphatic sprouts from each side of the dermal lymphatics, and quantified the ellipticity of 10 nuclei in $14.5 \mathrm{dpc}$ embryos in Figure $1 \mathrm{~J}, \mathbf{2 F}$ and $\mathbf{4 0}$. We quantified the number of branch points and lymphatic valves of the subcutaneous lymphatic network within $2000 \mu \mathrm{m}$ from the midline in $16.5 \mathrm{dpc}$ embryos for Figure 10. We quantified the orientation and nuclei ellipticity (nuclei length-to-width ratio) for the lymphatic valve nuclei that showed upregulated PROX1 (1.5 times of mean fluorescent intensity) for Figure $\mathbf{1 P}$ and $\mathbf{Q}$. We analysed 5 valves on the primary spokes of mesenteries from each embryo at $18.5 \mathrm{dpf}$ that were stained for F-Actin using Phalloidin, lymphatics using PROX1 and veins using EMCN. Since lymphatic valve cells have an upregulation of PROX1 (Srinivasan and Oliver, 2011), all Prox1 upregulated valve nuclei (1.5 times of mean fluorescent intensity) from each valve were quantified for nuclei ellipticity in Figure 1T. The intensity of F-Actin and PROX1 staining in Figure $1 \mathrm{U}$ was measured from the centre Z-section of each Z-stack through each valve based on the location of the nucleus. The ratio of valve to lymphatic vessel mean F-actin staining intensity was calculated. We selected 5 vessels from each side of the skin for the quantification of genetic interaction at a cellular level in Figure 2-4. We measured the width of the selected vessels at 10, 25, 50, $75,100 \mu \mathrm{m}$ from the tip of lymphatic sprouts, and the average width of the 5 measurements was considered as the width of the vessel. The number of LECs in the vessel tip were counted on 5 lymphatic sprouts on each side of the dermal lymphatics in Figure 4P. All quantifications were done genotype blinded.

\section{Statistical analysis}

We performed all statistical tests and generated all dot plots using GraphPad Prism 7 except for the rose diagram generated using PAST (Hammer et al., 2001). Specific tests used in each experiment are indicated in the figure legend. 


\section{Acknowledgements}

This work was supported by National Health and Medical Research Council of Australia (NHMRC) grants 1079158 and 1146352. BMH was supported by a fellowship (1155221) from the NHMRC. S.A.S. was supported by an NHMRC Program Grant (1053535), an NHMRC Senior Research Fellowship (1154746) and an NHMRC Investigator Grant (1176732) and by funds from the Operational Infrastructure Support Program provided by the Victorian Government,

We thank Dr. Kelly Betterman for their help with mesenteric lymphatic staining protocols and Prof. Peter Koopman for providing mice. All images were acquired at the Australian Cancer Research Foundation's Dynamic Imaging Facility at the Institute for Molecular Biosciences.

\section{Competing interests}

No competing interests declared.

\section{Funding}

NHMRC grants 1079158, 1146352, 1053535. NHMRC fellowships 1155221, 1154746, 1176732.

\section{Data availability}

No publicly available dataset used. 


\section{References}

Alders, M., Al-Gazali, L., Cordeiro, I., Dallapiccola, B., Garavelli, L., Tuysuz, B., Salehi, F., Haagmans, M.A., Mook, O.R., Majoie, C.B., et al. (2014). Hennekam syndrome can be caused by FAT4 mutations and be allelic to Van Maldergem syndrome. Hum Genet 133, 1161-1167.

Alva, J.A., Zovein, A.C., Monvoisin, A., Murphy, T., Salazar, A., Harvey, N.L., Carmeliet, P., and Iruela-Arispe, M.L. (2006). VE-Cadherin-Cre-recombinase transgenic mouse: A tool for lineage analysis and gene deletion in endothelial cells. Developmental Dynamics 235, 759-767.

Andre, P., Wang, Q., Wang, N., Gao, B., Schilit, A., Halford, M.M., Stacker, S.A., Zhang, X., and Yang, Y. (2012). The Wnt Coreceptor Ryk Regulates Wnt/Planar Cell Polarity by Modulating the Degradation of the Core Planar Cell Polarity Component Vangl2. J Biol Chem 287, 44518-44525.

Betterman, K.L., Sutton, D.L., Secker, G.A., Kazenwadel, J., Oszmiana, A., Lim, L., Miura, N., Sorokin, L., Hogan, B.M., Kahn, M.L., et al. (2020). Atypical cadherin FAT4 orchestrates lymphatic endothelial cell polarity in response to flow. The Journal of clinical investigation 130, 3315-3328.

Butler, M.T., and Wallingford, J.B. (2017). Planar cell polarity in development and disease. Nat Rev Mol Cell Biol 18, 375-388.

Buttler, K., Becker, J., Pukrop, T., and Wilting, J. (2013). Maldevelopment of dermal lymphatics in Wnt5a-knockout-mice. Developmental Biology 381, 365-376.

Cadigan, K.M., and Waterman, M.L. (2012). TCF/LEFs and Wnt Signaling in the Nucleus. Cold Spring Harb Perspect Biol 4, a007906-a007906.

Castelli, M., Boca, M., Chiaravalli, M., Ramalingam, H., Rowe, I., Distefano, G., Carroll, T., and Boletta, A. (2013). Polycystin-1 binds Par3/aPKC and controls convergent extension during renal tubular morphogenesis. Nature Communications 4 , 2658.

Cha, B., Geng, X., Mahamud, M.R., Fu, J., Mukherjee, A., Kim, Y., Jho, E.-H., Kim, T.H., Kahn, M.L., Xia, L., et al. (2016a). Mechanotransduction activates canonical Wnt/ $\beta$-catenin signaling to promote lymphatic vascular patterning and the development of lymphatic and lymphovenous valves. Genes Dev 30, 1454-1469.

Cha, B., Geng, X., Mahamud, M.R., Fu, J., Mukherjee, A., Kim, Y., Jho, E.H., Kim, T.H., Kahn, M.L., Xia, L., et al. (2016b). Mechanotransduction activates canonical Wnt/beta-catenin signaling to promote lymphatic vascular patterning and the development of lymphatic and lymphovenous valves. Genes Dev 30, 1454-1469.

Cha, B., Geng, X., Mahamud, M.R., Zhang, J.Y., Chen, L., Kim, W., Jho, E.-h., Kim, Y., Choi, D., Dixon, J.B., et al. (2018). Complementary Wnt Sources Regulate Lymphatic Vascular Development via PROX1-Dependent Wnt/ $\beta$-Catenin Signaling. Cell Rep 25, 571-584.e575.

Choi, D., Park, E., Jung, E., Seong, Y.J., Hong, M., Lee, S., Burford, J., Gyarmati, G., Peti-Peterdi, J., Srikanth, S., et al. (2017a). ORAI1 Activates Proliferation of Lymphatic Endothelial Cells in Response to Laminar Flow Through Krüppel-Like Factors 2 and 4. Circ Res 120, 1426-1439.

Choi, D., Park, E., Jung, E., Seong, Y.J., Yoo, J., Lee, E., Hong, M., Lee, S., Ishida, H., Burford, J., et al. (2017b). Laminar flow downregulates Notch activity to promote lymphatic sprouting. J Clin Invest 127, 1225-1240. 
Coxam, B., Sabine, A., Bower, Neil i., Smith, Kelly a., Pichol-Thievend, C., Skoczylas, R., Astin, Jonathan w., Frampton, E., Jaquet, M., Crosier, Philip s., et al. (2014). Pkd1 Regulates Lymphatic Vascular Morphogenesis during Development. Cell Reports 7, 623-633.

Francois, M., Caprini, A., Hosking, B., Orsenigo, F., Wilhelm, D., Browne, C., Paavonen, K., Karnezis, T., Shayan, R., Downes, M., et al. (2008). Sox18 induces development of the lymphatic vasculature in mice.(LETTERS)(Report). Nature 456, 643.

Hägerling, R., Pollmann, C., Andreas, M., Schmedt, C., Nurmi, H., Adams, R.H., Alitalo, K., Andresen, V., Kiefer, F., and Schulte-Merker, S. (2013). A novel multistep mechanism for initial lymphangiogenesis in mouse embryos based on ultramicroscopy. EMBO journal: European Molecular Biology Organization, 629-644. Halford, M.M., Armes, J., Buchert, M., Meskenaite, V., Grail, D., Hibbs, M.L., Wilks, A.F., Farlie, P.G., Newgreen, D.F., Hovens, C.M., et al. (2000). Ryk-deficient mice exhibit craniofacial defects associated with perturbed Eph receptor crosstalk. Nat Genet 25, 414-418.

Hammer, Ø., Harper, D.A.T., and Ryan, P.D. (2001). Past: Paleontological statistics software package for education and data analysis. Palaeontologia Electronica 4, XIXXX.

Hanaoka, K., Qian, F., Boletta, A., Bhunia, A.K., Piontek, K., Tsiokas, L., Sukhatme, V.P., Guggino, W.B., and Germino, G.G. (2000). Co-assembly of polycystin-1 and -2 produces unique cation-permeable currents. Nature 408, 990-994.

Hughes, J., Ward, C.J., Peral, B., Aspinwall, R., Clark, K., San Millán, J.L., Gamble, V., and Harris, P.C. (1995). The polycystic kidney disease 1 (PKD1) gene encodes a novel protein with multiple cell recognition domains. Nat Genet 10, 151-160.

James, J.M., Nalbandian, A., and Mukouyama, Y.-s. (2013). TGF $\beta$ signaling is required for sprouting lymphangiogenesis during lymphatic network development in the skin. Development 140, 3903-3914.

Karkkainen, M., Haiko, P., Sainio, K., Partanen, J., Taipale, J., Petrova, T., Jeltsch, M., Jackson, D., Talikka, M., Rauvala, H., et al. (2004). Vascular endothelial growth factor $\mathrm{C}$ is required for sprouting of the first lymphatic vessels from embryonic veins. Nature Immunology 5, 74-80.

Keeble, T.R., Halford, M.M., Seaman, C., Kee, N., Macheda, M., Anderson, R.B., Stacker, S.A., and Cooper, H.M. (2006). The Wnt receptor Ryk is required for Wnt5amediated axon guidance on the contralateral side of the corpus callosum. The Journal of neuroscience : the official journal of the Society for Neuroscience 26, 5840-5848.

Kim, S., Nie, H., Nesin, V., Tran, U., Outeda, P., Bai, C.-X., Keeling, J., Maskey, D., Watnick, T., Wessely, O., et al. (2016). The polycystin complex mediates Wnt/Ca2+ signalling. Nature Cell Biology 18, 752-764.

Koltowska, K., Betterman, K.L., Harvey, N.L., and Hogan, B.M. (2013). Getting out and about: the emergence and morphogenesis of the vertebrate lymphatic vasculature. Development 140, 1857-1870.

Kume, T. (2015). Lymphatic vessel development: fluid flow and valve-forming cells. J Clin Invest 125, 2924-2926.

Lu, W., Yamamoto, V., Ortega, B., and Baltimore, D. (2004). Mammalian Ryk Is a Wnt Coreceptor Required for Stimulation of Neurite Outgrowth. Cell 119, 97-108.

Lutze, G., Haarmann, A., Demanou Toukam, J.A., Buttler, K., Wilting, J., and Becker, J. (2019). Non-canonical WNT-signaling controls differentiation of lymphatics and extension lymphangiogenesis via RAC and JNK signaling. Scientific reports 9, 47394739. 
Macheda, M.L., Sun, W.W., Kugathasan, K., Hogan, B.M., Bower, N.I., Halford, M.M., Zhang, Y.F., Jacques, B.E., Lieschke, G.J., Dabdoub, A., et al. (2012). The Wnt Receptor Ryk Plays a Role in Mammalian Planar Cell Polarity Signaling. J Biol Chem 287, 29312-29323.

Nauli, S.M., Alenghat, F.J., Luo, Y., Williams, E., Vassilev, P., Li, X., Elia, A.E.H., Lu, W., Brown, E.M., Quinn, S.J., et al. (2003). Polycystins 1 and 2 mediate mechanosensation in the primary cilium of kidney cells. Nat Genet 33, 129-137.

Nims, N., Vassmer, D., and Maser, R.L. (2003). Transmembrane Domain Analysis of Polycystin-1, the Product of the Polycystic Kidney Disease-1 (PKD1) Gene: Evidence for 11 Membrane-Spanning Domains. Biochemistry 42, 13035-13048.

Oliver, G., and Srinivasan, R.S. (2010). Endothelial cell plasticity: how to become and remain a lymphatic endothelial cell. Development 137, 363-372.

Outeda, P., Huso, David L., Fisher, Steven A., Halushka, Marc K., Kim, H., Qian, F., Germino, Gregory G., and Watnick, T. (2014). Polycystin Signaling Is Required for Directed Endothelial Cell Migration and Lymphatic Development. Cell Rep 7, 634-644. Peters, D.J.M., and Sandkuijl, L.A. (1992). Genetic Heterogeneity of Polycystic Kidney Disease in Europe. Contributions to Nephrology, 128-139.

Pujol, F., Hodgson, T., Martinez-Corral, I., Prats, A.-C., Devenport, D., Takeichi, M., Genot, E., Mäkinen, T., Francis-West, P., Garmy-Susini, B., et al. (2017). Dachsous1Fat4 Signaling Controls Endothelial Cell Polarization During Lymphatic Valve Morphogenesis-Brief Report. Arterioscler Thromb Vasc Biol 37, 1732-1735.

Roy, J.P., Halford, M.M., and Stacker, S.A. (2018). The biochemistry, signalling and disease relevance of RYK and other WNT-binding receptor tyrosine kinases. Growth factors (Chur, Switzerland) 36, 15-40.

Sabine, A., Davis, M.J., Bovay, E., and Petrova, T.V. (2018). Characterization of Mouse Mesenteric Lymphatic Valve Structure and Function. Lymphangiogenesis 1846, 97-129.

Schindelin, J., Arganda-Carreras, I., Frise, E., Kaynig, V., Longair, M., Pietzsch, T., Preibisch, S., Rueden, C., Saalfeld, S., Schmid, B., et al. (2012). Fiji: an open-source platform for biological-image analysis. Nature Methods 9, 676.

Sörensen, I., Adams, R.H., and Gossler, A. (2009). DLL1-mediated Notch activation regulates endothelial identity in mouse fetal arteries. Blood 113, 5680-5688.

Srinivasan, R.S., and Oliver, G. (2011). Prox1 dosage controls the number of lymphatic endothelial cell progenitors and the formation of the lymphovenous valves. Genes \& development 25, 2187-2197.

Surya, V.N., Michalaki, E., Fuller, G.G., and Dunn, A.R. (2019). Lymphatic endothelial cell calcium pulses are sensitive to spatial gradients in wall shear stress. Mol Biol Cell 30, 923-931.

Tatin, F., Taddei, A., Weston, A., Fuchs, E., Devenport, D., Tissir, F., and Makinen, T. (2013). Planar Cell Polarity Protein Celsr1 Regulates Endothelial Adherens Junctions and Directed Cell Rearrangements during Valve Morphogenesis. Developmental Cell 26, 31-44.

Vassilev, P.M., Guo, L., Chen, X.-Z., Segal, Y., Peng, J.-B., Basora, N., Babakhanlou, H., Cruger, G., Kanazirska, M., Ye, C.-p., et al. (2001). Polycystin-2 Is a Novel Cation Channel Implicated in Defective Intracellular Ca2+ Homeostasis in Polycystic Kidney Disease. Biochemical and biophysical research communications 282, 341-350.

Wigle, J.T., and Oliver, G. (1999). Prox1 function is required for the development of the murine lymphatic system. Cell 98, 769-778.

Yang, Y., Garcia-Verdugo, J.M., Soriano-Navarro, M., Srinivasan, R.S., Scallan, J.P., Singh, M.K., Epstein, J.A., and Oliver, G. (2012). Lymphatic endothelial progenitors 
bud from the cardinal vein and intersomitic vessels in mammalian embryos. Blood 120, 2340-2348.

Yoder, B.K., Hou, X., and Guay-Woodford, L.M. (2002). The Polycystic Kidney Disease Proteins, Polycystin-1, Polycystin-2, Polaris, and Cystin, Are Co-Localized in Renal Cilia. J Am Soc Nephrol 13, 2508-2516. 


\section{FIGURE 1}
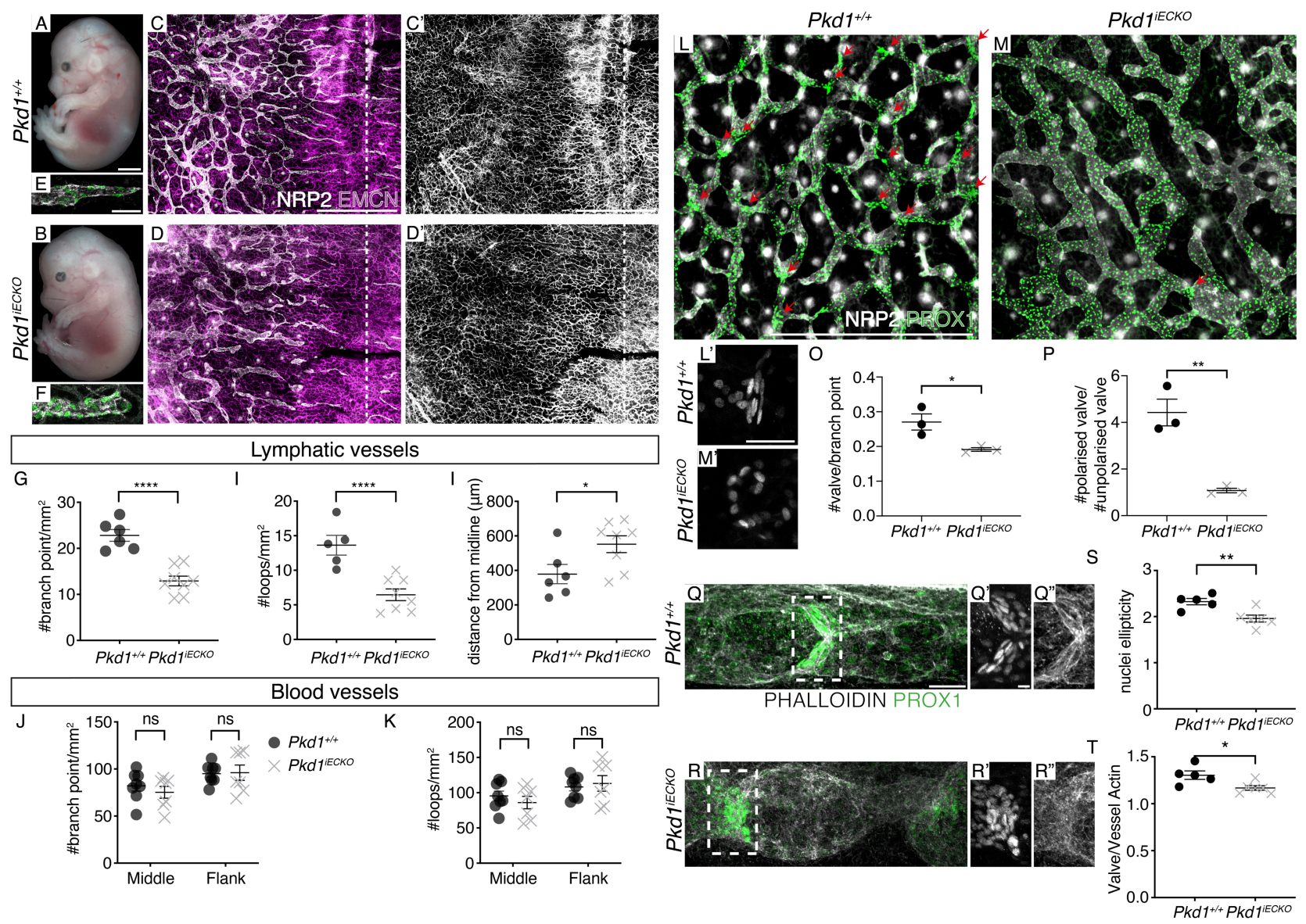

Figure 1. Pkd1 cell-autonomously regulates subcutaneous lymphatic network and lymphatic valve formation in mice.

(A-B) The overall morphology of $W t$ and $P k d 1^{i E C K O}$ mouse embryos at $14.5 \mathrm{dpc}$. Scale bar represents $2 \mathrm{~mm}$.

(C-D) Subcutaneous lymphatic networks in Wt and $P k d 1^{i E C K O}$ embryos at $14.5 \mathrm{dpc}$ stained with NRP2

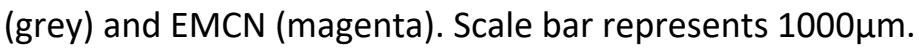

$\left(C^{\prime}-D^{\prime}\right)$ Subcutaneous blood vessels in $W t$ and $P k d 1^{i E C K O}$ embryos at $14.5 \mathrm{dpc}$ stained with EMCN. Scale bar represents $1000 \mu \mathrm{m}$.

(E-F) Subcutaneous lymphatic sprouts in Wt and Pkd1 $1^{\text {iECKO }}$ embryos at $14.5 \mathrm{dpc}$ stained with NRP2 (grey) and PROX1 (green). Scale bar represents $50 \mu \mathrm{m}$.

(G-J) Quantification of (G) the number of branch points and (H) loops per $\mathrm{mm}^{2}$, (I) distance of lymphatic sprouts from the midline $(\mu \mathrm{m})$, and $(J)$ nuclei ellipticity in $W t(n=6)$ and $P k d 1^{i E C K O}(n=8)$ embryos at $14.5 \mathrm{dpc}$. 
(K-L) Quantification of (K) the number of branch points and (L) loops per $\mathrm{mm}^{2}$ of the subcutaneous blood vessels in $W t(n=6)$ and Pkd1 ${ }^{\text {iECKO }}(\mathrm{n}=8)$ embryos at $14.5 \mathrm{dpc}$.

(M-N) Subcutaneous lymphatic networks in $W t$ and $P k d 1^{i E C K O}$ embryos at $16.5 \mathrm{dpc}$ stained with NRP2 (grey) and PROX1 (green). Scale bar represents $1000 \mu \mathrm{m}$. Arrows indicate lymphatic valves. $\left(\mathrm{M}^{\prime}-\mathrm{N}^{\prime}\right)$ Magnification of the mesenteric lymphatic valves in $W t$ and $P k d 1^{i E C K O}$ embryos at $18.5 \mathrm{dpc}$ with PROX1 staining. Scale bars represent $50 \mu \mathrm{m}$.

(O-Q) Quantification of $(O)$ the number of valves per branch point, $(P)$ ratio of polarised valves to unpolarised valves, and $(\mathrm{Q})$ nuclei ellipticity of valve LECs in $W t(\mathrm{n}=3)$ and $P k d 1^{i E C K O}(\mathrm{n}=3)$ embryos subcutaneous lymphatics at $16.5 \mathrm{dpc}$.

(R-S) Mesenteric lymphatic valves in Wt and $P k d 1^{i E C K O}$ embryos at $18.5 \mathrm{dpc}$ stained with Phalloidin (grey) and PROX1 (green). Scale bars represent 50 $\mathrm{mm}$.

$\left(R^{\prime}-S^{\prime \prime}\right)$ Magnification of the mesenteric lymphatic valves in $W t$ and $P k d 1^{i E C K O}$ embryos at $18.5 \mathrm{dpc}$ with $\left(R^{\prime}-S^{\prime}\right)$ PROX1 and ( $\left.R^{\prime \prime}-S^{\prime \prime}\right)$ Phalloidin staining. Scale bars represent $5 \mu \mathrm{m}$.

(T-U) Quantification for ( $T$ ) nuclei ellipticity and $(U)$ valve to vessel actin fluorescent intensity ratio in $W t(\mathrm{n}=5)$ and $P k d 1^{\text {iECKO }}(\mathrm{n}=6)$ embryos at $18.5 \mathrm{dpc}$.

Mean \pm s.e.m. are shown. Student's $t$-test. ${ }^{* * *}$ : p-value $<0.0001,{ }^{* * *}$ : p-value $<0.001,{ }^{* *}$ : pvalue $<0.01, *$ : $p$-value $<0.05$ 


\section{FIGURE 2}
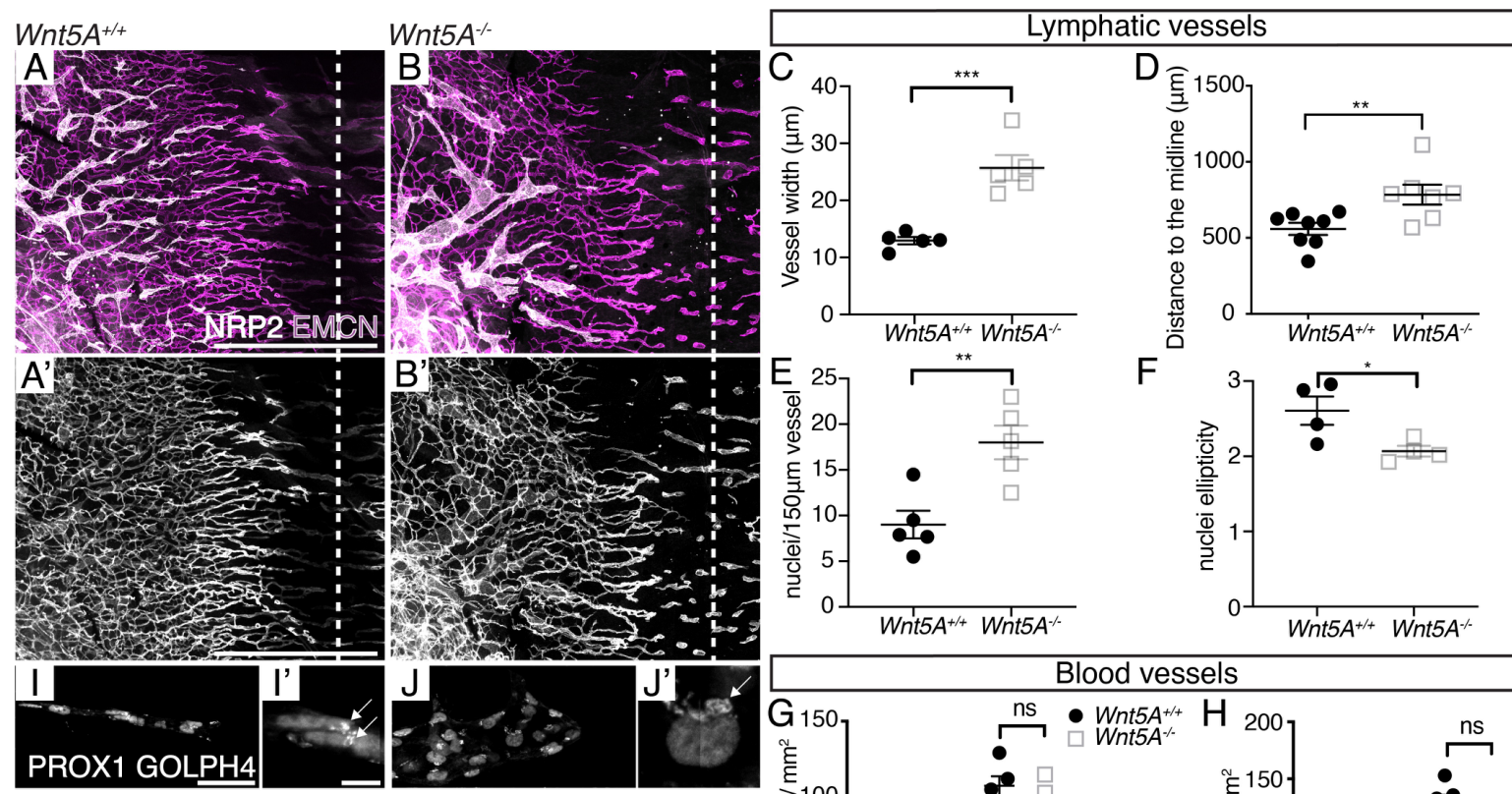

$\mathrm{K}$

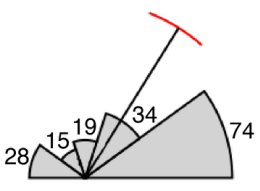

$\mathrm{L}$
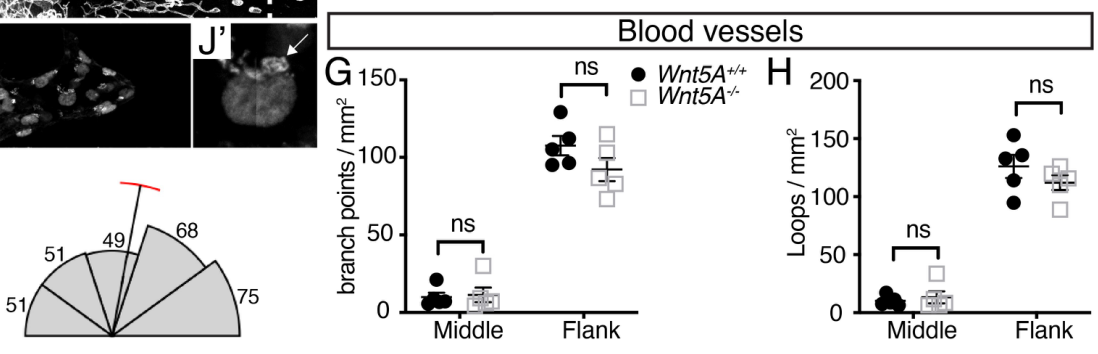

Figure 2. WNT5A mutant mice show subcutaneous lymphatic network and cell polarity defects

(A-B) Subcutaneous lymphatic networks in $W n t 5 a^{+/+}$and $W n t 5 a^{-/-}$mouse embryos at $13.5 \mathrm{dpc}$ stained with NRP2 (grey) and EMCN (magenta). Scale bar represents $1000 \mu \mathrm{m}$.

$\left(\mathrm{A}^{\prime}-\mathrm{B}^{\prime}\right)$ Subcutaneous blood vessel networks in $W n t 5 a^{+/+}$and $W n t 5 a^{-/}$mouse embryos at $13.5 \mathrm{dpc}$ stained with EMCN. Scale bar represents $1000 \mu \mathrm{m}$.

(C-F) Quantification of (C) vessel width, (D) distance from midline, (E) the number of nuclei/150 $\mu \mathrm{m}$ vessel, and (F) nuclei ellipticity of subcutaneous lymphatic vessel networks in Wnt5a $a^{+/+}(n=5)$ and Wnt $5 a^{-1-}(\mathrm{n}=5)$ embryos at $13.5 \mathrm{dpc}$.

(G-H) Quantification of ( $G$ ) the number of branch points and $(\mathrm{H})$ the number of loops of subcutaneous blood vessel networks in $W n t 5 a^{+/+}(n=5)$ and $W n t 5 a^{-/-}(n=5)$ embryos at $13.5 \mathrm{dpc}$.

(I-J') Subcutaneous lymphatic sprouts in $W n t 5 a^{+/+}$and $W n t 5 a^{-/-}$mouse embryos at $13.5 \mathrm{dpc}$ stained with PROX1 and GOLPH4. Scale bar represents $50 \mu \mathrm{m}$.

$\left(I^{\prime}-J^{\prime}\right)$ Magnification of a nucleus and its Golgi body in the stained subcutaneous lymphatic sprouts. Scale bar represents $5 \mu \mathrm{m}$.

(K-L) Quantification of Golgi body direction relative to the migration direction of lymphatic sprouts in Wnt5 $a^{+/+}(\mathrm{n}=5)$ and $W n t 5 a^{-/-}(\mathrm{n}=5)$ mouse embryos at $13.5 \mathrm{dpc}$.

Mean \pm s.e.m. are shown. Student's $t$-test. $* * * *$ : p-value<0.0001, ***: p-value<0.001, **: p-

value $<0.01, *$ : p-value $<0.05$ 


\section{FIGURE 3}

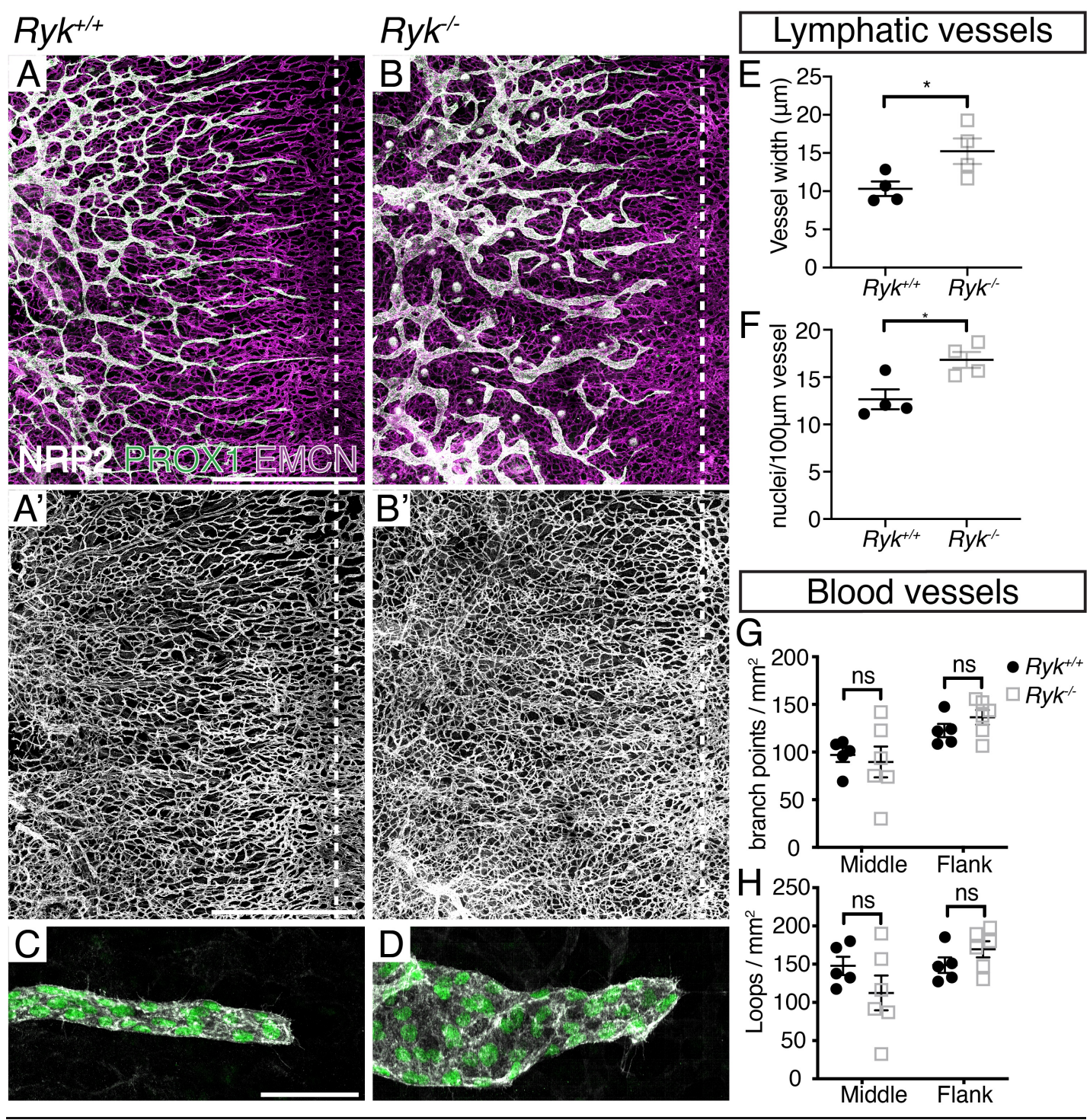

Figure 3. Ryk is required for subcutaneous lymphatic vascular morphogenesis

(A-B) Subcutaneous lymphatic networks in $R y k^{+/+}$and $R y k^{-/-}$mouse embryos at $14.5 \mathrm{dpc}$ stained with NRP2 (grey), PROX1 (green) and EMCN (magenta). Scale bar represents $1000 \mu \mathrm{m}$.

$\left(A^{\prime}-B^{\prime}\right)$ Subcutaneous blood vessel networks in $R y k^{+/+}$and $R y k^{-/}$mouse embryos at $14.5 \mathrm{dpc}$ stained with EMCN. Scale bar represents $1000 \mu \mathrm{m}$.

(C-D) Subcutaneous lymphatic sprouts in $R y k^{+/+}$and $R y k^{-/}$mouse embryos at $14.5 \mathrm{dpc}$ stained with NRP2 (grey) and PROX1 (green). Scale bar represents 50 $\mathrm{mm}$.

(E-F) Quantification of (E) vessel width and (F) the number of nuclei/100 $\mu \mathrm{m}$ vessel of subcutaneous lymphatic vessel networks in $R \mathrm{Kk}^{+/+}(\mathrm{n}=5)$ and $R \mathrm{Kk}^{-/}(\mathrm{n}=5)$ mouse embryos at $14.5 \mathrm{dpc}$.

(G-H) Quantification of (G) the number of branch points and (H) loops of subcutaneous blood vessel networks in $R y k^{+/+}(\mathrm{n}=5)$ and $R y k^{--}(\mathrm{n}=5)$ mouse embryos at $14.5 \mathrm{dpc}$.

Mean \pm s.e.m. are shown. Student's $t$-test. ${ }^{* * *}$ : p-value $<0.0001,{ }^{* * *}:$-value $<0.001,{ }^{* *}$ : p-

value $<0.01, *$ : $p$-value $<0.05$ 

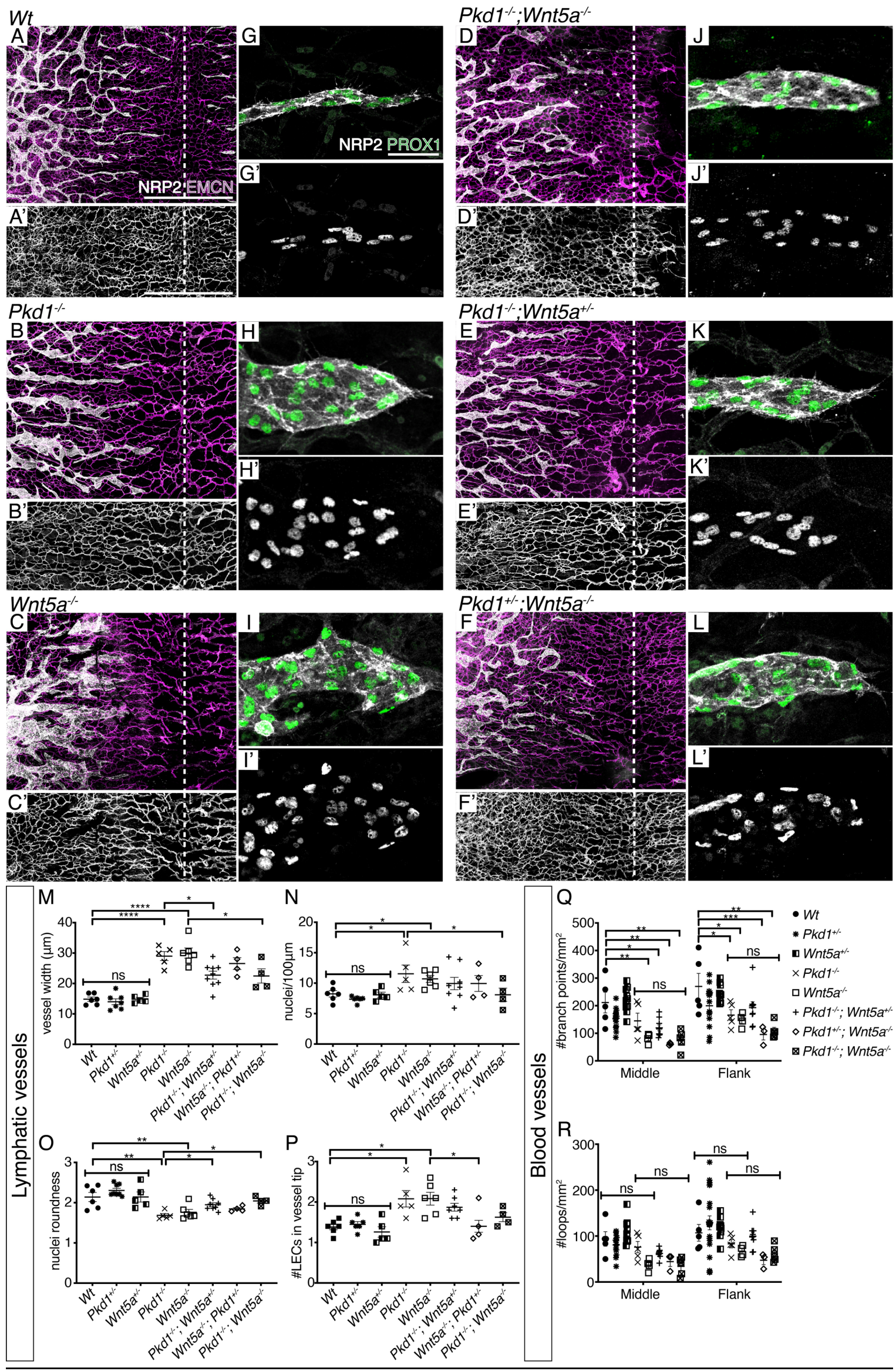
Figure 4. Pkd1 and WNT5A double mutants show less severe lymphatic defects than single mutants

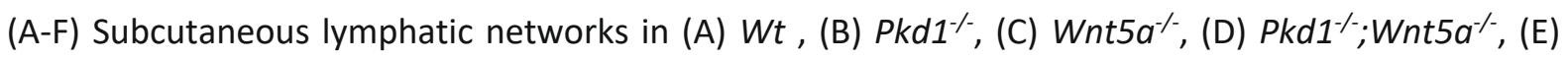

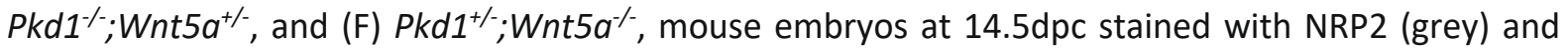
EMCN (magenta). Scale bar represents $1000 \mu \mathrm{m}$.

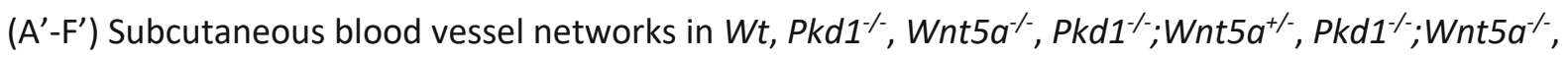
$P k d 1^{+\cdots} ; W n t 5 a^{--}, P k d 1^{-} ; W n t 5 a^{--}$mouse embryos at $14.5 \mathrm{dpc}$ stained with EMCN. Scale bar represents $1000 \mu \mathrm{m}$.

(G-L) Subcutaneous lymphatic sprouts in (G) Wt, (H) $P k d 1^{-\%}$, (I) Wnt5 $a^{-\%}$, (J) $P k d 1^{-\%}$;Wnt5a ${ }^{-\%}$, (K) Pkd1

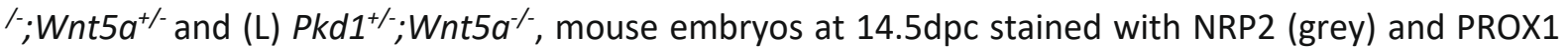
(green). Scale bar represents $50 \mu \mathrm{m}$.

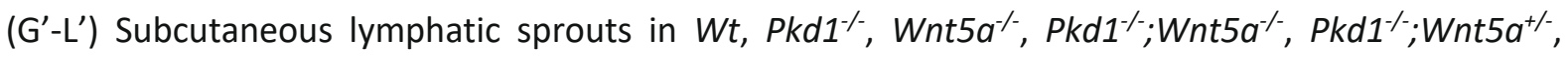
$P k d 1^{+/} ; W_{n t 5 a} \%$, mouse embryos at 14.5 dpc stained with PROX1.

(M-O) Quantifications of number of (M) vessel width, (N) the number of nuclei/100 $\mu \mathrm{m}$ vessel, (O)

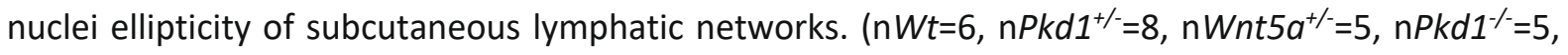

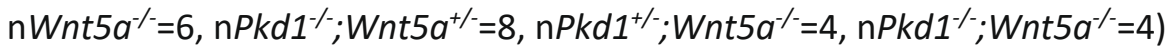

(P-Q) Quantifications of $(P)$ the number of branch points and (Q) loops per $\mathrm{mm}^{2}$ of subcutaneous blood vessel networks.

Mean \pm s.e.m. are shown. One-way ANOVA, Tukey's multiple comparisons test. ${ }^{* * * *}$ : p-value $<0.0001$, ***: p-value $<0.001,{ }^{* *}$ : p-value $<0.01, *$ : p-value $<0.05$ 